\title{
Talares del NE bonaerense con presencia de Ligustrum lucidum: Cambios en la estructura y la dinámica del bosque
}

\author{
M. Guadalupe Franco ${ }^{1, \bigotimes}$; Maia C. Plaza Behr²; Micaela Medina 2 ; Carolina Pérez ${ }^{2}$; \\ Ignacio A. Mundo ${ }^{13,3} ;$ J. Manuel Cellini ${ }^{2}$ \& Marcelo F. Arturi ${ }^{2}$ \\ ${ }^{1}$ Laboratorio de Dendrocronología e Historia Ambiental, Instituto Argentino de Nivología, Glaciología y Ciencias Ambientales \\ (IANIGLA) - CONICET. Mendoza, Argentina. ${ }^{2}$ Laboratorio de Investigación en Sistemas Ecológicos y Ambientales (LISEA), \\ Facultad de Ciencias Agrarias y Forestales, Universidad Nacional de La Plata. La Plata, Argentina. ${ }^{3}$ Facultad de Ciencias \\ Exactas y Naturales, Universidad Nacional de Cuyo. Mendoza, Argentina.
}

\begin{abstract}
RESUMEN. La presencia de nuevas especies arbóreas en ecosistemas boscosos desencadena modificaciones en la dinámica que se reflejan en su estructura. Estos procesos son especialmente relevantes desde el punto de vista de la conservación de bosques nativos. Los bosques de tala (Celtis ehrenbergiana var. ehrenbergiana) y coronillo (Scutia buxifolia) se consideran como una de las principales comunidades boscosas de la provincia de Buenos Aires y se encuentran protegidos en el marco de diversas reservas. En los talares de la Reserva El Destino, reconocidos entre los mejor conservados, se desarrolló un proceso de establecimiento de Ligustrum lucidum, especie que demuestra gran potencial invasor en otros bosques de la Argentina y del mundo. El objetivo de este trabajo fue caracterizar la estructura actual de estos talares e inferir los cambios en la dinámica vinculados con la presencia de esta especie. En la actualidad, estos bosques están dominados por ligustro, tanto en términos de densidad $(93 \%)$ como de área basal $(80 \%)$, y la estructura del componente nativo está lejos de los valores característicos. Cerca de $80 \%$ de los árboles nativos están muertos, valor muy superior al $20 \%$ de mortalidad natural, y se asocian de forma positiva con la densidad de ligustro. La exótica se instaló inicialmente en forma no selectiva y espacialmente aleatoria, y hoy llega a dominar el dosel al superar rápidamente en altura a las especies nativas. El estado actual se alcanzó en aproximadamente 20-25 años, lo cual implica un proceso de cambio estructural muy veloz. Estos resultados indican que estos talares cambiaron profundamente y, dado el grado de modificación, podrían concebirse como un sistema ecológico nuevo, sean ecosistemas noveles o híbridos. Cualquiera sea el enfoque, el control del ligustro y la restauración activa del componente nativo, así como la erradicación en áreas donde el establecimiento es reciente, resultan prioritarios para asegurar la perpetuidad de estos bosques.
\end{abstract}

[Palabras clave: bosques nativos, especies invasoras, cambios estructurales, Ecorregión Pampeana]

Aвstract. Talares from northeastern Buenos Aires in the presence of Ligustrum lucidum W. T. (Aiton): Changes in forest structure and dynamics. The presence of new tree species in forest ecosystems promotes changes in stand dynamics that are reflected by forest structure. These processes are particularly relevant in native forests conservation. Tala (Celtis ehrenbergiana var. ehrenbergiana) and coronillo (Scutia buxifolia) forests are considered among the main forest communities of Buenos Aires province, in Argentina, and are included in several protected areas. In the El Destino Reserve, a very well conserved native forest is currently in the presence of Ligustrum lucidum, which has demonstrated a high invasive potential in other forest of Argentina and the world. The aim of this work was to characterize present structure of these stands and to infer changes in the dynamics promoted by the presence of L. lucidum. We found that these talares are currently dominated by L. lucidum in tree density $(93 \%)$ and basal area $(80 \%)$, and that native forest structure has departed from the characteristic values. Around $80 \%$ of native trees are dead, widely surpassing the $20 \%$ reported natural mortality, and dead tree density is positively associated with L. lucidum density. The exotic species initially established in a non-selective and spatially random pattern and reached current canopy dominance by rapidly exceeding native species total height. Present conditions were reached approximately in 20-25 years, which indicate a high rate of structural change. These results demonstrate these talares have profoundly changed and, considering the degree of modification, could be treated as new ecological systems, either novel or hybrid ecosystems. Whichever the approach, the control of L. lucidum and the active restoration of the native component, as well as the eradication of the exotic species in areas where establishment is still recent, are all of high priority in order to ensure the long-term conservation of these forests.

[Keywords: native forests, invasive species, structural changes, Pampean Ecoregion]

Editor asociado: Guillermo Martínez Pastur 凶mgfranco@mendoza-conicet.gob.ar
Recibido: 28 de noviembre de 2017

Aceptado: 8 de junio de 2018 


\section{INTRODUCCIÓN}

El establecimiento de especies exóticas en bosques nativos implica modificaciones en la dinámica de dichas comunidades y, en consecuencia, desencadena cambios significativos en su estructura. Estas modificaciones se pueden reflejar desde el nivel individual, poblacional y comunitario como alteraciones en el crecimiento, la regeneración y la supervivencia de las especies nativas hasta el nivel ecosistémico, como variaciones en los ciclos del agua y los nutrientes (Leege and Murphy 2001). Por lo general, estos procesos fueron estudiados desde el enfoque de las invasiones, para lo cual se tomó en cuenta la proliferación y persistencia de estas especies como un proceso con consecuencias negativas en todos los niveles de organización (Mack 1996).

Si bien muchas especies vegetales fueron trasladadas hacia nuevos ambientes, sólo una pequeña proporción de ellas protagonizó procesos de invasión (Williamson and Fitter 1996; Mack et al. 2000). Sin embargo, representan una de las principales amenazas a la biodiversidad, a la estructura de las comunidades y a las funciones ecosistémicas (Wilcove et al. 1998; Kolar and Lodge 2001; Lee 2002), por lo que se les dedicó gran esfuerzo para determinar las condiciones que propician la ocurrencia de invasiones. Atributos tales como alta tasa de crecimiento, amplia plasticidad fenotípica, maduración reproductiva a temprana edad, producción de semillas pequeñas y en grandes cantidades, mecanismos eficientes de dispersión y capacidad de propagación asexual, fueron señalados comoaquellos que confieren ventajas a las invasoras, tanto para el establecimiento como para la competencia (Rejmánek and Richardson 1996; Alpert et al. 2000; Kolar and Lodge 2001; Petit et al. 2004; Lamarque et al. 2011). No obstante, las características que determinan el potencial invasor de las especies difieren entre ambientes; a su vez, estos ambientes son más o menos susceptibles a ser invadidos según su diversidad, régimen de disturbios y disponibilidad de recursos (Alpert et al. 2000; Petit et al. 2004; Lamarque et al. 2011).

Tradicionalmente, las invasiones biológicas fueron consideradas procesos negativos para los ecosistemas y, en consecuencia, las propuestas de manejo asociadas se concentraron en la exclusión o la eliminación de la especie invasora (Lodge et al. 2006). Sin embargo, en la actualidad se debate si los nuevos sistemas deben ser manejados para que recuperen sus características iniciales o existen aspectos positivos en $\mathrm{su}$ estado actual en términos de biodiversidad, servicios ecosistémicos o provisión de productos. Algunos autores resaltaron los beneficios que estos sistemas pueden presentar ante objetivos de conservación o restauración, en particular cuando se trata de sistemas muy alterados (Ewel and Putz 2004) en los que posiblemente la única opción viable sea considerar el manejo con permanencia de la exótica ( $\mathrm{D}^{\prime}$ Antonio and Meyerson 2002). Desde este punto de vista es posible considerar a las especies exóticas como componentes de ecosistemas noveles (Hobbs et al. 2006), con una dinámica propia. Estos nuevos sistemas pueden contribuir a la conservación de las especies nativas tanto en forma directa, mediante mecanismos de facilitación o como catalizadores de la sucesión en áreas degradadas, como en forma indirecta, manteniendo las funciones ecosistémicas y actuando, incluso, como proveedores alternativos de bienes y servicios (Otsamo 2000; D'Antonio and Meyerson 2002; Lugo 2004; Rodríguez 2006; Schlaepfer et al. 2011; Mascaro et al. 2012).

Ligustrum lucidum W.T. (Aiton) (ligustro), especie arbórea de origen asiático, fue introducida en diversas regiones con fines paisajísticos y demostró tener un gran potencial invasor en una variedad de ambientes en regiones como Australia, Nueva Zelanda y Estados Unidos (Cronk and Fuller 1995; Mowatt 1981; Panetta 2000; Wilcox 2000; Blanchon et al. 2011; Aslan et al. 2012). En la Argentina, el ligustro fue introducido a comienzos del siglo XX como planta ornamental (Rio and Achával 1904) y protagonizó procesos de invasión y exclusión competitiva de árboles nativos en sistemas muy diferentes como los bosques montanos de la región de las Yungas (Grau and Aragón 2000), el extremo sur de la selva paranaense en Punta Lara (Montaldo 1993), el monte serrano cordobés (Hoyos et al. 2010) y los talares del 
NE de la provincia de Buenos Aires (Ribichich and Protomastro 1998; Díaz Villa et al. 2016). En consecuencia, diversos efectos sobre las comunidades se documentaron en áreas ocupadas por esta especie, desde cambios en la estructura vertical, dominancia, diversidad y patrones de regeneración, reducción de crecimiento por competencia y simplificación de los estratos arbustivos y herbáceos, hasta modificaciones de hábitat con implicancias en la diversidad de aves (Lichstein et al. 2004; Hoyos et al. 2010; Ayup et al. 2014; Malizia et al. 2017). Entre sus características autoecológicas se destacan algunas que coinciden con rasgos usualmente asociados a la capacidad invasora: madurez temprana, gran producción de frutos, dispersión eficiente y rápida, crecimiento rápido, capacidad de rebrote y tolerancia a la sombra (Montaldo 1993; Grau and Aragón 2000; Aragón and Groom 2003; Lichstein et al. 2004; GavierPizarro et al. 2012). La disponibilidad de frutos maduros ocurre principalmente entre mayo y octubre (Montaldo 1993), que es la época de menor oferta de frutos carnosos por parte de las especies arbóreas nativas (Murriello et al. 1993); en consecuencia, esto podría conferir ventajas para la dispersión de ligustro. Una característica relevante es su capacidad de regenerarse y sobrevivir en ámbitos diferentes, desde pleno sol hasta bajo canopeos densos (Aragón and Groom 2003), lo que implica que su ingreso a los sistemas no se ve necesariamente asociado a disturbios $\mathrm{y}$, por lo tanto, el rango de ambientes en los que puede establecerse es amplio.

Los talares representan una de las comunidades boscosas más importantes de la Ecorregión Pampeana y, al igual que el resto de esa unidad, sufrieron un proceso intenso de degradación por actividades antrópicas tales como la ganadería y la extracción de leña y de materiales calcáreos del subsuelo (Arturi and Goya 2004). El reciente ordenamiento territorial bonaerense de bosques nativos declara una superficie de 969.000 ha de bosque bajo protección, la cual representa un $3 \%$ del territorio provincial (Ley $\mathrm{n}^{\mathrm{o}} 14.888 / 17$ ). Stupino et al. (2004) indicaron que los talares de la Reserva El Destino representan un área prioritaria deconservaciónenelParqueCostero del Sur en función de su extensa cobertura arbórea. En la actualidad, la expansión de L. lucidum es el agente modificador principal de la estructura y la dinámica de esos talares. Ribichich and Protomastro (1998) reportaron el inicio de este proceso en 1998; ellos encontraron regeneración avanzada de ligustro en bosques primarios y secundarios de tala y coronillo. Más recientemente, en la misma zona, Plaza Behr et al. (2016) estudiaron la efectividad de la remoción de ligustros adultos y la plantación de plantines de tala como método de restauración, y Diaz Villa et al. (2016) evaluaron la composición del banco de semillas en rodales con distinto grado de invasión. No obstante, no se encontraron reportes del efecto del establecimiento del ligustro en la estructura y dinámica de estos bosques.

El objetivo de este trabajo fue caracterizar la estructura actual de los talares en un sector con alta ocupación por ligustros e inferir modificaciones asociadas a su establecimiento en la dinámica estructural de los talares, entendida como los procesos de cambio en la estructura promovidos por la regeneración, el crecimiento y la mortalidad de árboles. En particular, se exploraron las relaciones entre el establecimiento de esta especie y los patrones de distribución diamétrica y mortalidad.

\section{Materiales y Métodos}

\section{Área de estudio}

Los talares se encuentran en la Ecorregión Pampeana (Morello et al. 2012), pero presentan una composición florística afín al Espinal (Torres Robles and Arturi 2009). Se extienden en áreas costeras desde el sur de Entre Ríos y Santa Fe hasta cerca de Mar del Plata, en la provincia de Buenos Aires (Cabrera 1976). Son comunidades boscosas que se desarrollan sobre cordones conchiles paralelos a la costa cuyas especies arbóreas dominantes son Celtis ehrenbergiana (Klotzsch) Liebm. var. ehrenbergiana (tala) y Scutia buxifolia Reissek (coronillo). Además, pueden hallarse Jodina rhombifolia (Hook. and Arn.) Reissek, Schinus longifolius (Lindl.) Speg., Sambucus australis Cham. and Schltdl. y Phytolacca dioica L. como especies acompañantes(Arturi and Goya 2004). 
El clima es templado, con bajas amplitudes térmicas diarias y estacionales, temperatura media anual entre $15^{\circ} \mathrm{C}$ y $17^{\circ} \mathrm{C}$ y precipitación anual entre 850 y $1065 \mathrm{~mm}$, con máximos de ocurrencia en otoño y primavera (Arturi and Goya 2004). Estos bosques representan una de las principales comunidades boscosas de la provincia de Buenos Aires (Goya et al. 1992). Luego de una historia de intenso uso antrópico (Parodi 1940), cobraron relevancia desde el punto de vista de la conservación y se encuentran protegidos en el marco de diversas reservas: Reserva Privada Campos del Tuyú (5 ha), Reserva Científica Otamendi (9 ha), Reserva Natural Isla Martín García (20 ha) y Reserva de Biosfera Parque Costero del Sur (MAB-UNESCO, 2363 ha), entre las que la última es la de mayor relevancia (Ribichich and Protomastro 1998). No obstante, el estado general de conservación de estos bosques se ve afectado por el uso histórico, que incluyó la extracción de madera para uso leñero y el desarrollo de la ganadería (Parodi 1940), actividades que alteraron su estructura, como también la extracción de conchilla, que implicó una remoción completa del bosque y alteró el ambiente en forma tal que resulta difícil su restauración (Plaza Behr et al. 2016).

Los talares de Magdalena y Punta Indio se caracterizan por presentar un dosel entre 7 y $11 \mathrm{~m}$, densidades entre 800 y 2000 individuos/ha y una estructura con ramificación basal abundante (Arturi and Goya 2004), consecuencia de la tendencia al rebrote de las especies dominantes y asociada al uso histórico de extracción de leña en estos bosques. No obstante, su persistencia en la actualidad indicaría que en el pasado no hubo un gran interés en el desmonte, probablemente por la rentabilidad reducida de su corta y comercialización como leña, y a que no se haya percibido como un obstáculo para las actividades realizadas (Arturi et al. 2006). La actual reserva de flora y fauna autóctonas El Destino ( $35^{\circ} 8^{\prime \prime} \mathrm{S}-57^{\circ} 23^{\prime \prime} \mathrm{O}$ ), ubicada en el partido de Magdalena, ha funcionado como resguardo de los ambientes naturales desde mediados del siglo XX, cuando cerca de 500 ha de la estancia fueron excluidas del uso ganadero para ser destinadas a la conservación de la flora y fauna regional. A partir de 1984, la reserva pasó a integrar la Reserva de Biosfera "Parque Costero del Sur", cuyos objetivos se asocian con la investigación, la formación y difusión con interés en la conservación y el desarrollo sostenible; funciona como modelo regional que los promueva (Arturi et al. 2009). En las 274 ha de talares que alberga la Reserva El Destino (Cagnoni et al. 1995) se encuentran los rodales mejores conservados dentro de la Reserva de Biosfera (CEPA 1989) y allí se realizaron los muestreos correspondientes al bosque mixto de Goya et al. (1992). Hoy en día, esos talares presentan un estado avanzado de colonización por ligustro.

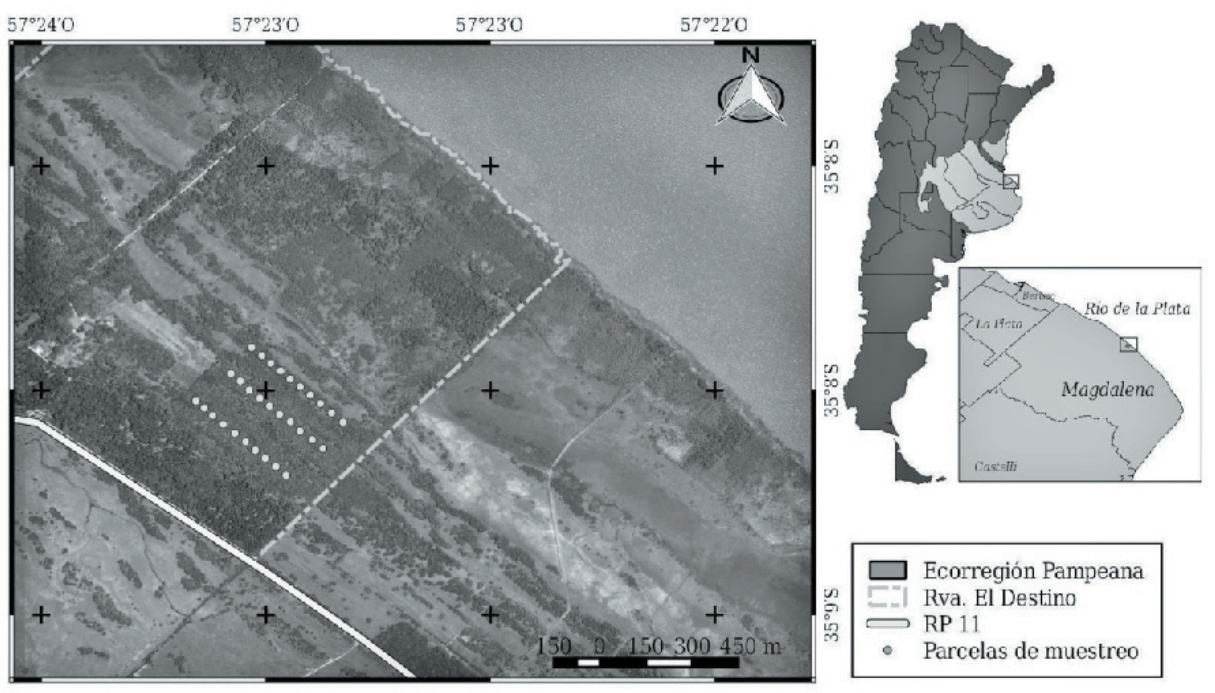

Figura 1. Ubicación de la Ecorregión Pampeana y de la Reserva El Destino. Los puntos indican los centroides de las parcelas.

Figure 1. Location of the Pampean Ecoregion and El Destino Reserve. Dots indicate plot's centroids. 


\section{Muestreo y análisis de datos}

Para caracterizar la estructura de la Reserva El Destino se realizó un muestreo en talares con presencia de ligustro a lo largo de tres cordones (Figura 1). En cada cordón se distribuyeron 10 parcelas circulares de $100 \mathrm{~m}^{2}$ cada $30 \mathrm{~m}$, a lo largo del eje central de cada cordón. En las parcelas se registró el diámetro a $1.3 \mathrm{~m}$ (DAP), la altura total y la especie y la condición (vivo o muerto) de todos los individuos arbóreos, definidos como aquellos cuyo DAP fuera igual o mayor a $5 \mathrm{~cm}$.

Para caracterizarlos estructuralmente se definieron dos grupos: especies nativas y $L$. lucidum, y se los discriminó según su condición. A su vez, se consideró al total de nativas (vivas + muertas) como indicador de la situación del bosque nativo antes del establecimiento de la exótica. Se calculó la densidad de árboles (individuos/ha) y área basal $\left(\mathrm{m}^{2} / \mathrm{ha}\right.$ ) correspondiente a cada subgrupo y al total de nativas, y se caracterizaron gráficamente las distribuciones diamétricas para los dos grupos de especies.

Con el fin de identificar patrones espaciales de establecimiento y mortalidad se analizó la existencia de patrones de distribución mediante la razón varianza/media del número de árboles por parcela (Ludwig and Reynolds 1988), de manera separada para ligustro y nativas, vivos y muertos. Este cociente tiene el valor 1 para una distribución de Poisson que corresponde a distribuciones aleatorias de los árboles y resulta mayor o menor que 1 para distribuciones agregadas o regulares respectivamente. Se evaluó este índice para nativas muertas, total de nativas, ligustros vivos y ligustros mayores a $20 \mathrm{~cm}$ de DAP (esta última categoría se definió como indicadora de los individuos establecidos más tempranamente). La significancia de los alejamientos respecto de la distribución aleatoria se evaluó a través de la prueba de chi-cuadrado (Ludwig and Reynolds 1988).

A su vez, se exploraron las relaciones de asociación entre los árboles nativos y $L$. lucidum mediante análisis de correlación de Pearson. Este análisis se llevó a cabo, en primer lugar, entre la densidad de nativas muertas y la densidad total de ligustro para evaluar en qué medida la mortalidad de nativas ocurrió preferencialmente en las parcelas con mayor densidad de ligustro. En segundo lugar, la correlación se calculó tomando la densidad total de nativas y ligustros mayores a $20 \mathrm{~cm}$ de DAP para evaluar la existencia de una relación entre la densidad del bosque nativo y el establecimiento de ligustro. Por último, se analizaron las diferencias entre nativas y L. lucidum en la relación DAP-altura mediante el ajuste del modelo de Chapman-Richards (Richards 1959) entre ambas variables.

\section{RESUltados}

Entre los árboles vivos, las especies nativas representaron un $7 \%$ de la densidad y $20 \%$ del área basal (Tabla 1). Del estrato nativo, $80 \%$ de los individuos y $70 \%$ del área basal a)

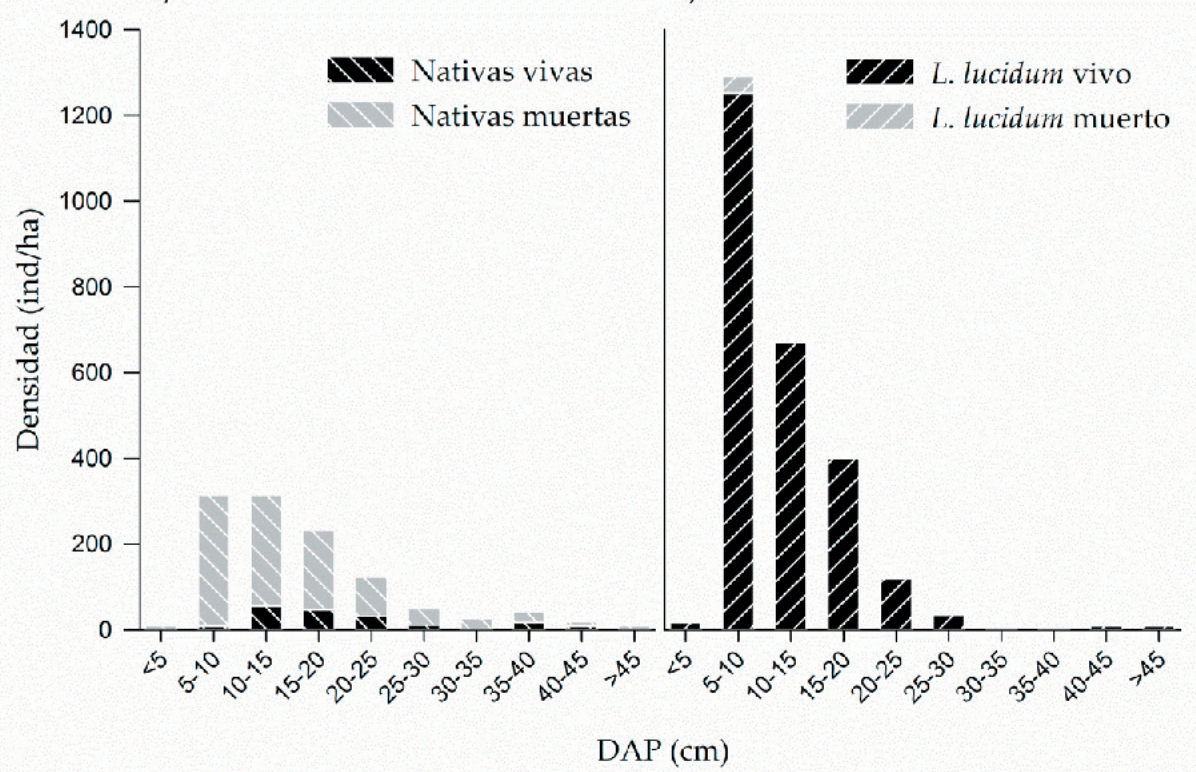

Figura 2. Estructura diamétrica de especies nativas (a) y L. lucidum (b).

Figure 2. Diametric structure of native species (a) and $L$. lucidum (b). 
Tabla 1. Parámetros estructurales de especies arbóreas nativas y L. lucidum: densidad de individuos ( $\delta$, individuos/ha), área basal $\left(\mathrm{AB}, \mathrm{m}^{2} / \mathrm{ha}\right) \mathrm{y}$ altura total $(\mathrm{HT}, \mathrm{m})$.

Table 1. Structural parameters of native tree species and L. lucidum: tree density ( $\delta$, individuals/ha), basal area $(\mathrm{AB}$, $\mathrm{m}^{2} / \mathrm{ha}$ ) and total height (HT, m).

\begin{tabular}{|c|c|c|c|c|c|c|c|c|c|c|c|c|}
\hline & \multicolumn{7}{|c|}{ Nativas } & \multicolumn{5}{|c|}{ L. lucidum } \\
\hline & \multicolumn{2}{|c|}{ Muertas } & \multicolumn{3}{|c|}{ Vivas } & \multicolumn{2}{|c|}{ Total } & \multicolumn{2}{|c|}{ Muertos } & \multicolumn{3}{|c|}{ Vivos } \\
\hline & $\delta$ (ind/ha) & $\begin{array}{c}\mathrm{AB} \\
\left(\mathrm{m}^{2} / \mathrm{ha}\right)\end{array}$ & $\delta$ (ind/ha) & $\mathrm{AB}\left(\mathrm{m}^{2} / \mathrm{ha}\right)$ & $\begin{array}{l}\mathrm{HT} \\
(\mathrm{m})\end{array}$ & $\delta$ (ind/ha) & $\begin{array}{c}\mathrm{AB} \\
\left(\mathrm{m}^{2} / \mathrm{ha}\right)\end{array}$ & $\delta$ (ind/ha) & $\begin{array}{c}\mathrm{AB} \\
\left(\mathrm{m}^{2} / \mathrm{ha}\right)\end{array}$ & $\delta$ (ind/ha) & $\begin{array}{c}\mathrm{AB} \\
\left(\mathrm{m}^{2} / \mathrm{ha}\right)\end{array}$ & $\begin{array}{l}\text { HT } \\
\text { (m) }\end{array}$ \\
\hline Media & 743 & 19.1 & 190 & 7.5 & 5.3 & 933 & 26.6 & 40 & 0.7 & 2520 & 31.6 & 9.5 \\
\hline $\mathrm{DE}$ & 307 & 11.7 & 320 & 11.8 & 5 & 423 & 14.6 & 84 & 2.0 & 1133 & 10.2 & 0.8 \\
\hline Error & 115 & 4 & 119 & 4 & & 158 & 5 & 31 & 1 & 423 & 4 & \\
\hline Error \% & 0.15 & 0.23 & 0.63 & 0.59 & & 0.17 & 0.21 & 0.78 & 1.08 & 0.17 & 0.12 & \\
\hline
\end{tabular}

Estadísticos: media, desvío estándar (DE), error y error porcentual (Error \%)

a)

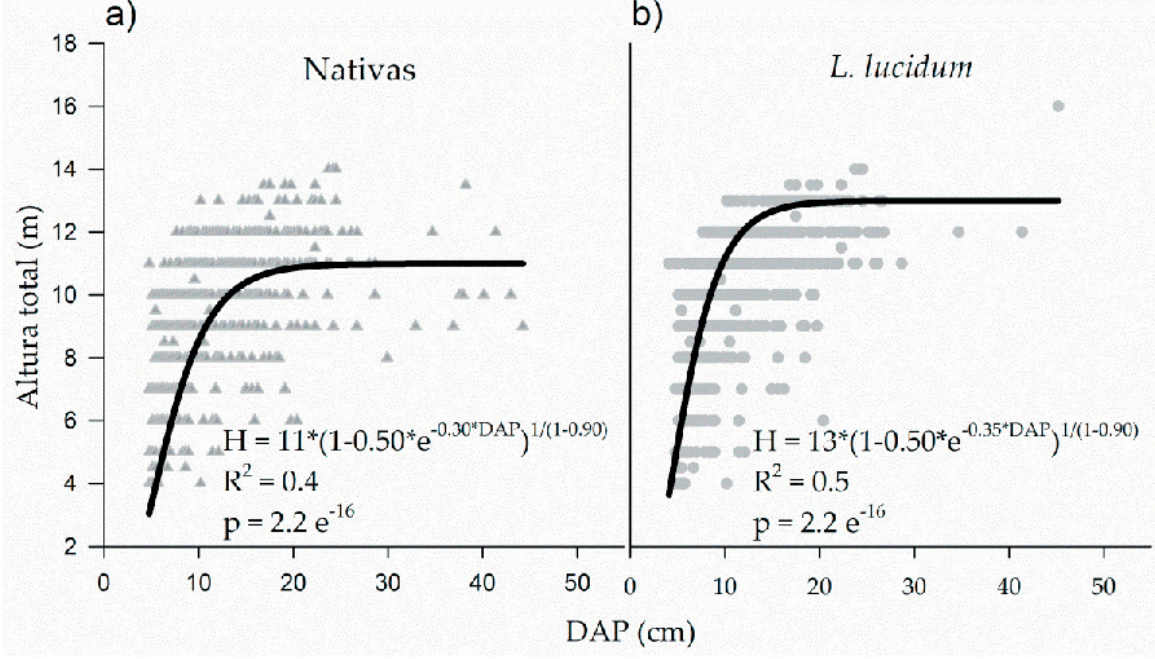

Figura 3. Relación entre la altura total y el diámetro a la altura del pecho (DAP) y función asociada en especies nativas (a) y L. lucidum (b).

Figure 3. Total height - diameter at breast height (DAP) relation and associated function for native species (a) and L. lucidum (b). correspondieron a árboles muertos. La distribución diamétrica del total de nativas presentó diámetros máximos de $\sim 45 \mathrm{~cm}$ y una mayor densidad de individuos menores de $20 \mathrm{~cm}$ de DAP, mientras que los individuos muertos tendieron a mostrar mayor proporción en las clases menores de $20 \mathrm{~cm}$ (Figura 2). L. lucidum presentó una distribución similar en términos de rango, aunque con mayor proporción de individuos menores de $15 \mathrm{~cm}$, que se asimiló claramente a una distribución de " $j$ " invertida. Los ligustros vivos fueron más abundantes que el total de nativas; superó más de dos veces su densidad y 20\% su área basal. Los ligustros muertos representaron sólo el 2\% del total de la especie.

La razón varianza/media presentó valores superiores a 1 tanto para nativas (3.2 para muertas, 3.4 para nativas totales) como para ligustro vivo (4.1), lo que indicó una distribución agrupada. Para ligustros de DAP mayor a $20 \mathrm{~cm}$, este índice fue igual 0.9, lo cual indicó una distribución aleatoria.
El análisis de correlación entre densidad de individuos demostró independencia entre la densidad de nativas y ligustros mayores a 20 $\mathrm{cm}(\mathrm{r}=-0.24, P>0.280)$. Sin embargo, se encontró una relación positiva entre densidad de nativas muertas y ligustro ( $\mathrm{r}=0.42, P<0.047)$.

La altura promedio de L. lucidum fue 9.5 $\mathrm{m}$, mientras que en las nativas alcanzó sólo $5.3 \mathrm{~m}$ (Tabla 1). Los modelos de relación alturaDAP indicaron que ligustro alcanzó $10 \mathrm{~m}$ de altura con DAP cercano a $10 \mathrm{~cm}$, mientras que los árboles nativos alcanzaron esa altura con DAP de alrededor de $15 \mathrm{~cm}$ (Figura 3).

\section{DISCUSIÓN}

La estructura y la dinámica del componente nativo se encuentran modificadas respecto de lo esperado para talares en esta zona. $\mathrm{Su}$ estructura original, representada por la densidad y el área basal de árboles nativos totales, se ubica dentro del rango definido por Arturi y Goya (2004) para los talares del 
partido de Magdalena, con valores levemente menores a los encontrados por Goya et al. (1992) para el bosque codominado por tala y coronillo. Asimismo, la distribución diamétrica dominada por las clases menores y la distribución espacial agrupada de los individuos también coinciden con lo reportado por Goya et al. (1992). Sin embargo, cerca del $80 \%$ de los árboles nativos se encuentran muertos, lo que indica un proceso de mortalidad avanzado muy superior al correspondiente a la dinámica natural de los talares, que representa alrededor de $20 \%$ (Goya et al. 1992).

Actualmente, el bosque está dominado por un dosel de ligustro que supera en altura al estrato arbóreo nativo y lo excede en densidad y área basal. El cambio hacia la estructura actual se inició con un establecimiento no selectivo de la exótica respecto de la estructura del bosque nativo, como lo indica la distribución aleatoria de los ligustros de mayor DAP y la falta de correlación entre su densidad y la de nativas. Luego, el posterior desarrollo del ligustro se puede asociar al proceso de mortalidad de nativas, lo cual se evidencia mediante la correlación positiva encontrada entre las densidades de nativas muertas y de la exótica, de acuerdo con lo esperado para un proceso competitivo. La mencionada situación permite explicar la elevada mortalidad de las nativas como consecuencia del sombreado que provoca una exclusión competitiva por parte de la exótica al alcanzar el dosel, tal como ocurre con otros bosques con presencia de ligustro (Hoyos et al. 2010).

La relación observada entre el establecimiento de ligustro y la mortalidad de especies nativas se asemeja a los procesos desencadenados por esta misma especie en otros bosques de la Argentina y del mundo. Lichstein et al. (2004) encontraron una dominancia deligustro de $80 \%$ en área basal y escasa proporción de nativas grandes vivas en fragmentos montanos de las Yungas invadidos por esta especie. Asimismo, en bosques chaqueños de las Sierras Chicas de Córdoba se reportó que en los rodales en los que ligustro fue la especie más abundante hubo una elevada mortalidad de nativas, por lo que los estadíos avanzados de invasión se catalogaron como críticos (Hoyos et al. 2010). En ese mismo trabajo se identificó un gradiente de estructuras asociadas a diferentes etapas del proceso de invasión, vinculadas principalmente con las densidades de ligustros mayores, ligustros jóvenes y nativas muertas, y se señaló que en los casos de invasión más avanzada sólo sobrevivían los individuos de mayor tamaño de la nativa Lithraea molleoides (Vell.) Engl. (molle). De forma similar, en bosques australianos invadidos por esta especie sólo sobreviven individuos grandes de Eucalyptus sp. L'Hér. (Swarbrick et al. 1999), mientras que el estrato arbustivo y de árboles bajos queda dominado por ligustro (Mowatt 1981). No obstante, existen otras situaciones en las que esta especie no logra comportarse como invasora, como en el caso de los pastizales pampeanos, donde las condiciones ambientales impiden su establecimiento (Mazia et al. 2001) o en los bosques urbanos de Japón, donde sus similitudes con las especies nativas limitan sus efectos invasivos a un máximo de codominancia (Lei et al. 2016).

Al considerar la situación actual de estos talares conjuntamente con los antecedentes se visualiza la velocidad con que ocurrió este proceso de cambio en la dominancia. La presencia de ligustro no fue reportada por Goya et al. (1992) en su relevamiento de individuos mayores de $5 \mathrm{~cm}$ de DAP en el mismo sitio de estudio, aunque encontraron abundantes renovales de $\sim 2 \mathrm{~m}$ de altura de esta especie bajo el dosel nativo en 1990, cuando llevaron a cabo sus muestreos (Goya y Arturi, comunicación personal). Al menos para el 2016, cuando se relevaron los datos analizados en este trabajo, ya se había alcanzado la estructura actual. Esto evidencia la rapidez de crecimiento y dispersión del ligustro en este ambiente. En un muestreo realizado entre 2016 y 2017 en el sector estudiado (Plaza Behr et al., datos no publicados) se encontró que el máximo incremento diamétrico ocurre en los ligustros de 10 a $15 \mathrm{~cm}$ de DAP, de los cuales $20 \%$ se aproxima a $1 \mathrm{~cm} /$ año. Esos valores resultan coherentes con la antigüedad de los registros y con la proporción elevada de individuos menores de $20 \mathrm{~cm}$ de DAP. Malizia et al. (2017) reportaron valores similares para ligustros creciendo en bosques secundarios de las Yungas, donde el incremento promedio alcanzó $0.69 \mathrm{~mm} /$ año. Sin embargo, estos autores no encontraron aumento en la mortalidad de nativas en parcelas con 20 años de presencia de ligustro, y la abundancia de la exótica alcanzó únicamente $4 \%$ de densidad y área basal, lo cual indica una dinámica de cambio diferente a la que ocurre en los talares. Considerando que el ligustro se establece sin dificultades en ambos ambientes, es probable que las diferencias en los ritmos de cambio se deban a las diferentes características de las comunidades nativas que implican el establecimiento de diferentes relaciones entre 
las especies nativas y la exótica. Además, cabe destacar que la Reserva El Destino fue mantenida sin pastoreo vacuno o con una carga muy baja desde mediados de la década del 80 , lo que podría haber facilitado el establecimiento de esta especie, ya que sus renovales son consumidos por el ganado (Tecco et al. 2007).

Como se expuso anteriormente, la velocidad con que se alcanzó el estado actual de dominancia es consecuencia del crecimiento rápido de esta especie. Si se toma en cuenta el DAP promedio y máximo actual del componente exótico y el tiempo transcurrido, se puede estimar una tasa de crecimiento diamétrico del orden de $1 \mathrm{~cm} /$ año, con un máximo de $3 \mathrm{~cm} /$ año, que coincide con los crecimientos reportados por Aragón y Groom (2003) para renovales y árboles de esta especie en diferentes condiciones de luminosidad. A su vez, su capacidad de dominar el dosel se evidencia al analizar las tendencias en altura, que sugieren un crecimiento temprano en altura del ligustro en comparación con las nativas, lo que queda en evidencia por la diferente relación entre la altura y el DAP. Asimismo, los resultados encontrados resultan coincidentes respecto a la falta de selectividad del ligustro para el establecimiento, que puede regenerar con éxito independientemente de las condiciones iniciales del bosque nativo (Aragón and Groom 2003; Aragón and Morales 2003).

En contraste, la regeneración de las especies dominantes de los talares se encuentra limitada por factores competitivos con los árboles adultos incluso en el bosque libre de ligustro (Arturi and Goya 2004). Es probable que el establecimiento de ligustro refuerce esos efectos sin que su propia regeneración se vea limitada, como lo sugiere su distribución diamétrica y la abundancia de individuos menores de $5 \mathrm{~cm}$ de DAP (Arturi, observación personal). En consecuencia, se puede afirmar que L. lucidum se comporta como invasora en los talares y, debido a las ventajas competitivas que tiene sobre las especies arbóreas nativas, la dinámica desencadenada conduce a una situación crítica, donde la comunidad tiende a cambiar hacia la dominancia de la exótica y la exclusión de las nativas.

Si se considera además que esta especie se estableció en las áreas mejor preservadas de la Reserva de Biosfera Parque Costero del Sur (Goya et al. 1992; Stupino et al. 2004), este proceso resulta muy relevante desde el punto de vista de la conservación de estos bosques; Esto resalta la necesidad de contar con propuestas de manejo que contribuyan a asegurar la perpetuidad de los talares. Se reportó que cuando el proceso de establecimiento se encuentra muy avanzado, como en este caso, la erradicación resulta muy difícil (Aragón and Groom 2003), por lo que el manejo de estos rodales se podría enfocar desde la perspectiva de los ecosistemas noveles. Este enfoque implica reconocer al bosque con ligustro como un nuevo sistema con funciones ecológicas propias, que podría estar sujeto a tratamientos silvícolas que permitan 1) reducir las condiciones de competencia que impiden el desarrollo de las especies nativas y 2) generar recursos madereros sobre la base de la extracción de ligustro. No obstante, un enfoque intermedio podría identificar aún alguna posibilidad de retornar al sistema anterior, concibiendo a este ecosistema como híbrido (Hobbs et al. 2014), en el que las acciones tenderían a un fin último de restauración, pero asociado con un proceso no focalizado sólo en la dicotomía de restauración/erradicación de especies.

Sin embargo, cualquiera de estas propuestas requiere reconocer los rasgos del sistema y sus componentes que limitan o potencian la posibilidad de intervención. En cuanto al ligustro, dadas las características de su madera, la producción de leña (Wilcox 2000) o de madera para construcción de viviendas (Benetti 2015) son destinos posibles que resultan compatibles con su velocidad de crecimiento y capacidad de rebrote. Por su parte, la regeneración de las especies nativas en estos bosques es limitada, por lo cual su repoblación se debería basar sobre propuestas de restauración activa tales como la plantación de las especies características, evaluada recientemente por Plaza Behr et al. (2016). A su vez, cualquier estrategia de estas características para las áreas más afectadas por el establecimiento de ligustro debería ser acompañada por acciones para evitar su propagación hacia otros sectores de los talares de la reserva, considerando su capacidad de rápida expansión (Gavier-Pizarro et al. 2012). Asimismo, la erradicación en áreas donde el establecimiento es reciente debería ser una prioridad, atendiendo a la estrecha ventana temporal donde esta actividad es una opción factible, que en estos bosques no supera los 20 años. No obstante, la perspectiva hacia el futuro indica que aun cuando se pudiera controlar el ligustro y se lograra reincorporar a las leñosas nativas, los sectores más afectados 
de los talares han cambiado profundamente y representan un sistema ecológico nuevo.

AgradecimiEnTos. Este trabajo fue financiado por la Facultad de Ciencias Agrarias y Forestales de la UNLP. Agradecemos a la Fundación Elsa Shaw de Pearson por apoyar la investigación en la Reserva El Destino y a los estudiantes de Ingeniería Forestal y de la Facultad de Ciencias Naturales y Museo (UNLP) que colaboraron con los muestreos en el marco de las asignaturas Ecología Forestal y Ecología y Manejo de Bosques Nativos, respectivamente. También agradecemos a los revisores anónimos del trabajo por sus valiosos comentarios y sugerencias.

\section{REFERENCIAS}

Alpert, P., E. Bone, and C. Holzapfel. 2000. Invasiveness, invasibility and the role of environmental stress in the spread of non-native plants. Perspectives in Plant Ecology, Evolution and Systematics 3(1):52-66.

Aragón, R., and M. Groom. 2003. Invasion by Ligustrum lucidum (Oleaceae) in NW Argentina: Early stage characteristics in different habitat types. Revista de Biología Tropical 51(1):59-70.

Aragón, R., and J. M. Morales. 2003. Species composition and invasion in NW Argentinian secondary forests: Effects of land use history, environment and landscape. Journal of Vegetation Science 14:195-204.

Arturi, M. F., and J. F. Goya. 2004. Estructura, dinámica y manejo de los talares del NE de Buenos Aires (Capítulo 10). Pp. 1-23 en M. F. Arturi, J. L. Frangi and J. F. Goya (eds.). Ecología y manejo de los bosques de Argentina. Publicación multimedia. Editorial de la Universidad Nacional de La Plata. Buenos Aires, Argentina.

Arturi, M. F., C. A. Pérez, M. Horlent, J. F. Goya, and S. Torres Robles. 2006. El manejo de los talares de Magdalena y Punta Indio como estrategia para su conservación. Pp. 37-45 en E. Mérida and J. Athor (eds.). Talares bonaerenses y su conservación. Fundación de Historia Natural Félix de Azara. Buenos Aires.

Arturi, M. F., M. Pérez Meroni, C. Paleo, and R. Herrera. 2009. Lineamientos para una zonificación del Parque Costero del Sur basada en la relación del paisaje con la cultura. Páginas 17-36 en J. Athor (ed.). Parque Costero del Sur Naturaleza, conservación y patrimonio cultural. Fundación de Historia Natural Félix de Azara. Buenos Aires.

Aslan, C. E., M. Rejmánek, and R. Klinger. 2012. Combining efficient methods to detect spread of woody invaders in urban-rural matrix landscapes: an exploration using two species of Oleaceae. Journal of Applied Ecology 49: 331-338.

Ayup, M. M., L. Montti, R. Aragón, and H. R. Grau. 2014. Invasion of Ligustrum lucidum (Oleaceae) in the southern Yungas: Changes in habitat properties and decline in bird diversity. Acta Oecologica 54:72-81.

Benetti, F. 2015. Estudio de la durabilidad natural y de las propiedades tecnológicas de la madera de Ligustrum lucidum W. T. Aiton. Tesis de grado. Universidad Nacional de La Plata.

Blanchon D., J. Pusateri, M. Galbraith, and S. Thorpe. 2011. Sampling indigenous ground-beetles in a stand of nonnative tree privet (Ligustrum lucidum) in New Zealand raises new management questions. Ecological Management and Restoration 12(3):234-236.

Cabrera, A. L. 1976. Regiones fitogeográficas argentinas Enciclopedia Argentina de Agricultura y Jardinería. 2da edición, tomo 1, Ed. ACME. Buenos Aires. Pp. 85.

Cagnoni, M., A. Faggi, and A. M. Ribichich. 1995. Carta de vegetación de la Reserva 'El Destino' (Pcia. Buenos Aires, Argentina). Contribuciones científicas de la Sociedad Argentina de Estudios Geográficos 7:21-28.

CEPA (Centro de Estudios y Proyectos del Ambiente). 1989. Parque Costero del Sur. UNESCO, Argentina.

Cronk, Q., and J. Fuller. 1995. Plant Invaders: The threat to natural ecosystems. Chapman and Hall. London. UK. Pp. 241.

D'Antonio, C., and L. A. Meyerson. 2002. Exotic Plant Species as Problems and Solutions in Ecological Restoration: A Synthesis. Restoration Ecology 10(4):703-713.

Díaz Villa, M. V. E. D., N. Madanes, P. M. Cristiano and, G. Goldstein. 2016. Composición del banco de semillas e invasión de Ligustrum lucidum en bosques costeros de la provincia de Buenos Aires, Argentina. Bosque 37(3):581-590.

Ewel, J. J., and F. E. Putz. 2004. A place for alien species in ecosystem restoration. Frontiers in Ecology and the Environment 2(7):354-360.

Gavier-Pizarro, G. I., T. Kuemmerle, L. E. Hoyos, S. I. Stewart, C. D. Huebner, N. S. Keuler, and V. C. Radeloff. 2012. Monitoring the invasion of an exotic tree (Ligustrum lucidum) from 1983 to 2006 with Landsat TM/ETM+ satellite data and Support Vector Machines in Córdoba, Argentina. Remote Sensing of Environment 122:134-145.

Goya J. F., G. Placci, M. F Arturi, and A. Brown. 1992. Distribución y características estructurales de los Talares de la reserva de biosfera "Parque Costero del Sur". Revista de la Facultad de Agronomía, La Plata 68(1):53-64.

Grau, H. R., and R. Aragón. 2000. Arboles invasores de la sierra de San Javier, Tucumán, Argentina. Pp. 5-20 en H. R. Grau and R. Aragón (eds.). Ecología de árboles exóticos en las Yungas argentinas. LIEY-UNT, Tucumán, Argentina.

Hobbs, R. J., S. Arico, J. Aronson, J. S. Baron, P. Bridgewater, V. A. Cramer, P. R. Epstein, J. J. Ewel, C. A. Klink, A. E. Lugo, D. Norton, D. Ojima, D. M. Richardson, E. W. Sanderson, F. Valladares, M. Vilà, R. Zamora, and M. Zobel. 2006. Novel ecosystems: theoretical and management aspects of the new ecological world order. Global Ecology and Biogeography 15:1-7.

Hobbs, R. J., E. Higgs, C. M. Hall, P. Bridgewater, F. S. Chapin III, E. C. Ellis, J. J. Ewel, L. M. Hallet, J. Harris, K. B. 
Hulvey, S. T. Jackson, P. L. Kennedy, C. Kueffer, L. Lach, T. C. Lantz, A. E. Lugo, J. Mascaro, S. D. Murphy, C. R. Nelson, M. P. Perring, D. M. Richardson, T. R. Seastedt, R. J. Standish, B. M. Starzomski, K. N. Suding, P. M. Tognetti, L. Yakob, and L. Yung. 2014. Managing the whole landscape: historical, hybrid, and novel ecosystems. Frontiers in Ecology and the Environment 12(10):557-564.

Hoyos, L. E., G. I. Gavier-Pizarro, T. Kuemmerle, E. H. Bucher, V. C. Radeloff, and P. A. Tecco. 2010. Invasion of glossy privet (Ligustrum lucidum) and native forest loss in Sierras Chicas of Córdoba, Argentina. Biological Invasions 12: 3261-3275.

Kolar, C. S., and D. M. Lodge. 2001. Progress in invasion biology: predicting invaders. Trends in Ecology and Evolution 16(4):199-204.

Lamarque, J. L., S. Delzon, and C. J. Lortie. 2011. Tree invasions: a comparative test of the dominant hypotheses and functional traits. Biological Invasions 13:1969-1989.

Lee, C. E. 2002. Evolutionary genetics of invasive species. Trends in Ecology and Evolution 17(8):9-11.

Leege, L. M., and P. G. Murphy. 2001. Ecological effects of the non-native Pinus nigra on sand dune communities. Canadian Journal of Botany 79:429-437.

Lei, T., N. Nishikawa, and N. Yamashita. 2016. Park pruning prompts a competitive reversal of an exotic tree, Ligustrum lucidum, in urban forests of Japan. Urban Ecosystems 19(3):1103-1118.

Ley N ${ }^{\circ}$ 14.888. 2017. Ley de Conservación y Manejo de Bosques Nativos. Ordenamiento Territorial. Provincia de Buenos Aires.

Lichstein, J. W., H. R. Grau, and R. Aragón. 2004. Recruitment limitation in secondary forests dominated by an exotic tree. Journal of Vegetation Science 15(6):721-728.

Lodge, D. M., S. Williams, H. J. Mac Isaac, K. R. Hayes, B. Leung, S. Reichard, R. N. Mack, P. B. Moyle, M. Smith, D. A. Andow, J. T. Carlton, and A. McMichael. 2006. Biological invasions: recommendations for U.S. policy and management. Ecological Applications 16(6):2035-2054.

Ludwig, J. A., and J. F. Reynolds. 1988. Statistical Ecology: A Primer on Methods and Computing. John Wiley and Sons, New York, USA. Pp. 354.

Lugo, A. E. 2004. The outcome of alien tree invasions in Puerto Rico. Frontiers in Ecology and the Environment 2(5): 265-273.

Mack, R. N. 1996. Predicting the identity and fate of plant invaders: emergent and emerging approaches. Biological Conservation 78:107-121.

Mack, R. N., D. Simberloff, W. M. Lonsdale, H. Evans, M. Clout, and F. Bazzaz. 2000. Biotic invasions: causes, epidemiology, global consequences, and control. Ecological Applications 10(3):689-710.

Mascaro, J., R. F. Hughes, and S. A. Schnitzer. 2012. Novel forests maintain ecosystem processes after the decline of native tree species. Ecological Monographs 82(2):221-228.

Mazia, N. C., E. J. Chaneton, C. M. Ghersha, and R. J. León. 2001. Limits to tree species invasions in pampean grassland and forest plant communities. Oecologia 128:594-602.

Malizia, A., O. Osinaga-Acosta, P. A. Powell, and R. Aragón. 2017. Invasion of Ligustrum lucidum (Oleaceae) in subtropical secondary forests of NW Argentina: declining growth rates of abundant native tree species. Journal of Vegetation Science 28:1240-1249.

Montaldo, N. H. 1993. Dispersión por aves y éxito reproductivo de dos especies de Ligustrum (Oleaceae) en un relicto de selva subtropical en la Argentina. Revista Chilena de Historia Natural 66:75-85.

Mowatt, J. 1981. Control of large-leaved privet (Ligustrum lucidum) and small-leaved privet (L. sinense) in urban bushland. Pp. 165-168 en Proceedings of the Sixth Australian Weeds Conference. Vol 1.

Otsamo, R. 2000. Secondary forest regeneration under fast-growing forest plantations on degraded Imperata cylindrica grasslands. New Forests 19:69-93.

Panetta, F. D. 2000. Fates of fruits and seeds of Ligustrum lucidum W. T. Ait. and L. sinense Lour. maintained under natural rainfall or irrigation. Australian Journal of Botany 48:701-705.

Parodi, L. R. 1940. La distribución geográfica de los talares en la Provincia de Buenos Aires. Darwiniana 4:33-56.

Plaza Behr, M. C., C. A. Pérez, J. F. Goya, M. Azcona, and M. F. Arturi. 2016. Plantación de Celtis ehrenbergiana como técnica de recuperación de bosques invadidos por Ligustrum lucidum en los talares del NE de Buenos Aires. Ecología Austral 26:171-177.

Petit, J., R. Bialozyt, and P. Garnier-Géré. 2004. Ecology and genetics of tree invasions: from recent introductions to Quaternary migrations. Forest Ecology and Management 197:117-137.

Rejmánek, M., and D. M. Richardson. 1996. What attributes make some plant species more invasive? Ecology 77(6): 1655-1661.

Ribichich, A. M., and J. Protomastro. 1998. Woody vegetation structure of xeric forest stands under different edaphic site conditions and disturbance histories in the Biosphere Reserve "Parque Costero del Sur", Argentina. Plant Ecology 139:189-201.

Richards, F. J. 1959. A flexible growth function for empirical use. Journal of Experimental Botany 10(2):290-300.

Rio, M. E., and L. Achaval (eds.). 1904. Geografía de la Provincia de Córdoba. Volumen 1. Compañía Sudamericana de Billetes de Banco. Pp. 569.

Rodríguez, L. F. 2006. Can invasive species facilitate native species? Evidence of how, when, and why these impacts occur. Biological Invasions 8:927-939. 
Schlaepfer, M. A., D. F. Sax, and J. D. Olden. 2011. The potential conservation value of non-native species. Conservation Biology 25(3):428-437.

Stupino, S. A., M. F. Arturi, and J. L. Frangi. 2004. Estructura del paisaje y conservación de los bosques de Celtis tala Gill ex Planch del NE de la provincia de Buenos Aires. Revista de la Facultad de Agronomía 105(2):37-45.

Swarbrick, J. T., S. M. Timmins, and K. M. Bullen. 1999. The biology of Australian weeds. 36. Ligustrum lucidum Aiton and Ligustrum sinense Lour. Plant Protection Quarterly 14(4):122-130.

Tecco, P. A., D. E. Gurvich, N. Pérez-Harguindeguy, M. Cabido, and G. A. Bertone. 2007. Facilitation and interference underlying the association between the woody invaders Pyracantha angustifolia and Ligustrum lucidum. Applied Vegetation Science 10(2):211-218.

Torres Robles, S. S., and M. F. Arturi. 2009. Variación de la composición y riqueza florística en los talares del Parque Costero del Sur y su relación con el resto de los talares bonaerenses. Pp. 104-121 en J. Athor (ed.). Parque Costero del Sur: Magdalena y Punta Indio, provincial de Buenos Aires. Fundación de Historia Natural Félix de Azara. Buenos Aires.

Wilcove, D. S., D. Rothstein, J. Dubow, A. Phillips, and E. Losos. 1998. Quantifying threats to imperiled species in the United States. BioScience 48(8):607-615.

Wilcox, M. 2000. Tree privet (Ligustrum lucidum) - a controversial plant. Auckland Botanical Society Journal 55(2): 72-74.

Williamson, M., and A. Fitter. 1996. The varying success of invaders. Ecology 77(6):1661-1666. 